\title{
Weakly Anisotropic Noncentrosymmetric Superconductors with Radial Line Nodes and the Origin of the Anomalous Thermodynamic Data
}

\author{
Mehmet Günay ${ }^{1}$, Tuğrul Hakioğlu ${ }^{2,3}$, and Hasan Hüseyin Sömek ${ }^{3}$ \\ ${ }^{1}$ Department of Physics, Bilkent University, 06800 Ankara, Turkey \\ ${ }^{2}$ Consortium of Quantum Technologies in Energy (Q-TECH), Energy Institute, Istanbul Technical University, \\ 34469 Istanbul, Turkey \\ ${ }^{3}$ Department of Physics, Istanbul Technical University, 34469 Istanbul, Turkey
}

(Received November 4, 2016; accepted January 19, 2017; published online February 27, 2017)

In noncentrosymmetric superconductors (NCSs), the inversion symmetry (IS) is most commonly broken by an antisymmetric spin-orbit coupling (SOC). Removing the spin degeneracy and splitting the Fermi surface (FS) into two branches. A two component condensate is then produced mixing an even singlet and an odd triplet. When the triplet and the singlet strengths are comparable, the pair potential can have rich nodes. The angular line nodes (ALNs) are associated with the point group symmetries of the anisotropic lattice structure and they are widely studied in the literature. When the anisotropy is weak, other types of nodes can be present which then affect differently the low temperature properties. Here, we focus on the weakly anisotropic NCSs and the line nodes which survive in the limit of full isotropy. We study the topology of these radial line nodes (RLNs) and show that it is characterized by the $Z_{2}$ index similar to the quantum-spin-Hall Insulators. From the thermodynamic perspective, the RLNs cause, even in the topological phases, an exponentially suppressed low temperature behaviour which can be mistaken by nodeless $s$-wave pairing, thus, providing an explanation to a number of recent experiments with contraversial pairing symmetries. In the rare case when the RLN is on the Fermi surface, the exponential suppression is replaced by a linear temperature dependence. The RLNs are difficult to detect, and for this reason, they may have escaped experimental attention. We demonstrate that Andreev conductance measurements with clean interfaces can efficiently identify the weakly anisotropic (WA) conditions where the RLNs are expected to be found.

\section{Introduction}

Superconducting symmetries beyond the conventional BCS spin singlet state were known since 1960s. Distinct examples are ${ }^{3} \mathrm{He},{ }^{1)}$ heavy fermion, ${ }^{2)}$ high $T_{\mathrm{c}}{ }^{3)}$ superconductors as well as the NCSs. ${ }^{4,5)}$ Strongly momentum dependent electronic correlations, broken spin-degeneracy, broken IS and the SOC add to the variety of factors yielding exotic spin and momentum dependent phenomena leading to the formation of unconventional Cooper pairs. ${ }^{6-8)}$ The manifested or broken time reversal symmetry (TRS) ${ }^{9)}$ and the non trivial topologies in the electronic bands add to the plethora that make the full understanding an experimental and theoretical challenge. ${ }^{10)}$

The NCSs break the IS while a substantial part of all superconductors has this symmetry. The IS is broken along an axis, let's say $z$, most commonly by the presence of a SOC and the superconducting electrons are confined in the homogeneous $x-y$ plane with the wavectors $\mathbf{k}=\left(k_{x}, k_{y}\right)=$ $k(\cos \phi, \sin \phi)$. We associate a vector $\mathbf{G}=\alpha\left(-k_{y}, k_{x}, 0\right)$ with the SOC where $\alpha$ is the SOC constant. The parity and the spin are not conserved individually, i.e., the OPs accommodate an even singlet $\psi_{\mathbf{k}}$ and an odd triplet $\mathbf{d}_{\mathbf{k}}=\left(d_{x \mathbf{k}}, d_{y \mathbf{k}}, d_{z \mathbf{k}}\right)$ simultaneously. The full $2 \times 2$ pair potential in the spinor basis is $\Delta(\mathbf{k})=i\left(\psi_{\mathbf{k}}+\mathbf{d}_{\mathbf{k}} \cdot \sigma\right) \sigma_{y}$ and the connection with the spin basis is made by the Eq. (5). We consider the TRS and the Pauli exchange symmetries to be manifest and therefore $\psi_{\mathbf{k}}$ and $\mathbf{d}_{\mathbf{k}}$ are real.

The smoking gun of the unconventional pairing in an NCS is the nodes of the pair potential ${ }^{4,11-13)}$ and a better understanding of the nodal structure can provide unique evidence on the pairing interaction. Our work here is concentrated on the type of nodes and the degree of anisotropy. In particular we wish to explore the nodes in the WA conditions when the lattice point group symmetries are not the rulers in the symmetries of the OPs. We therefore use an anisotropic expansion of the OPs in the angular momentum- $L_{z}$ basis as $X_{\mathbf{k}}=\sum_{m} X_{\mathbf{k}}^{(m)}$ where $X_{\mathbf{k}}^{(m)}=$ $\mathcal{Y}_{m}(\hat{\mathbf{k}}) X_{k}^{(m)}, \hat{\mathbf{k}}=\mathbf{k} / k$ and $\mathcal{Y}_{m}(\hat{\mathbf{k}}) \propto(\cos m \phi, \sin m \phi)$ are the basis functions of $L_{z}$ with eigenvalue $m$ describing the anisotropy ${ }^{14)}$ with $X_{\mathbf{k}}=\left(\psi_{\mathbf{k}}, \mathbf{d}_{\mathbf{k}}\right)$. Here $X_{k}^{(m)}=\left(\psi_{k}^{(m)}, \mathbf{d}_{k}^{(m)}\right)$ are radial functions of $k$.

Under the strongly anisotropic (SA) conditions, the point nodes can occur at the TRS points $\mathbf{k}=0$ and, in tetragonal symmetry at $\mathbf{k}=\{( \pm \pi, 0),(0, \pm \pi)\}$. Angular line nodes (ALNs) can also be present along $k_{x}= \pm k_{y}$ or $\mathbf{k}=\left(0, k_{y}\right)$ or $\left(k_{x}, 0\right)$. In low temperatures, ALNs are evidenced by integer exponents in the temperature dependence of the specific heat, the London penetration depth, the heat conductivity, the ultrasound attenuation and this has been observed in a number of cases ${ }^{11,12)}$ among which are the celebrated TRS preserving $\mathrm{CePt}_{3} \mathrm{Si}$ and the TRS breaking $\mathrm{Sr}_{2} \mathrm{RuO}_{4}$. Other experiments also exist where ALNs cannot explain the thermodynamic data. ${ }^{14)}$ Despite a large number of experimental and theoretical work, a one-to-one understanding between the temperature exponents and the nodes is missing.

In tetragonal symmetry, the leading terms in the angular momentum expansion are usually considered as the $s(m=0)$ and the $d$-wave $(m=2)$ components of the singlet $\psi_{\mathbf{k}} \simeq$ $\psi_{k}^{(0)}+\psi_{k}^{(2)} \cos 2 \phi$, and the $p$ - and the $f$-wave components of the triplet $\mathbf{d}_{\mathbf{k}} \simeq \mathbf{D}_{\mathbf{k}}\left(d_{k}^{(0)}+d_{k}^{(2)} \cos 2 \phi\right)$ where, for WA systems, $\mathbf{D}_{\mathbf{k}}$ in our work here is a vector in the $x-y$ plane (see Sect. 2) describing the orientation of the triplet component. The mixed state pairing potential $\Delta_{\mathbf{k}}^{\lambda}=\psi_{\mathbf{k}}-\lambda \gamma_{k} F_{\mathbf{k}}$, where $\lambda= \pm$ is the band splitting due to the broken IS, is directly responsible for opening an energy gap at the Fermi level as well as giving rise to a topological band structure. Here, 
$F_{\mathbf{k}}=\left|\mathbf{d}_{\mathbf{k}}\right|$ and $\gamma_{k}$ is a function of $k$ which can only take the values \pm 1 (see Sect. 2). In our case, the $\Delta_{\mathbf{k}}^{\lambda}$ is given by

$$
\Delta_{\mathbf{k}}^{\lambda}=A_{k}^{\lambda}+B_{k}^{\lambda} \cos 2 \phi,
$$

where $A_{k}^{\lambda}=\psi_{k}^{(0)}-\lambda \gamma_{k} F_{k}^{(0)}, B_{k}^{\lambda}=\psi_{k}^{(2)}-\lambda \gamma_{k} F_{k}^{(2)}$ are $m=0,2$ components respectively. The ALNs are obtained for $\left|A_{k}^{\lambda} / B_{k}^{\lambda}\right| \leq 1$ in the small wavelength limit where the anisotropy is pronounced. In this regime the effect of the short range electronic correlations in the form of SDWs, CDWs or Fermi surface nesting is important. On the other hand, in the other (WA) case the nodes are dominated by the first term in Eq. (1) where they are closed lines encircling the origin.

The strong or weak anisotropy is therefore determined by the leading term in Eq. (1) being $B_{k}$ or $A_{k}$ respectively. Since the magnitude of the second term is limited by $\left|B_{k}\right|$, we use a simple model where $B_{k}=\epsilon$ is constant and $A_{k}$ is given by the profile in Fig. 1(a). The change from the fully isotropic RLNs to the SA ALNs can then be studied as $\epsilon$ is changed. This transmutation changes the rotational symmetry of the energy gap yielding a variety which can yield a number of other intermediate solutions including nodal arcs and point nodes [see Fig. 1(b) for an example]. These features can be revealed in real systems where $A_{k}$ and $B_{k}$ are self-consistently determined. In this context, a superconducting node engineering may be developed in the future which can provide valuable information about the elusive mechanisms behind the unconventional pairing and, not the least new and exotic device applications.

The RLNs in the pair potential occur at sharp radial positions $k=k_{\Delta}$. There, the singlet and the triplet acquire equal strengths, i.e., $\left|\psi_{k_{\Delta}}\right|=\left|F_{k_{\Delta}}\right|$, and their positions depend on the specific non-uniform $k$-dependence of these OPs which can extend well beyond the Fermi surface (see above). A full handling of the momentum dependence is therefore necessary. ${ }^{15)}$ For Hubbard like short range correlations, the effective interaction can be unstable near AFM/FM transitions where the momentum-dependence of the spin-charge susceptibilities are crucial for the triplet state. ${ }^{4,10)}$ On the other hand, effective dielectric screening must be added when long range Coulomb interaction or phonon exchange is studied. ${ }^{16)}$ The line nodes can exist in grossly different scales of anisotropy [as shown for instance in Fig. 1(b)] providing us tools in understanding the general mechanisms behind the unconventional pairing.

It is commonly stated in the literature that, the WA conditions is a signature of the lack of strong correlations leading to the s-wave pairing and trivial topology. On the other hand, the pairing mechanisms and particularly the role of the strong correlations are not well understood. It is known that, strong electronic correlations observed in some NCSs (such as the magnetic fluctuations in heavy fermions) are not crucial in many other NCSs. For instance sesquicarbides $R_{2} \mathrm{C}_{3-x}$ where $R$ is a rare-earth element, the ternary $\mathrm{Li}_{2}\left(\mathrm{Pd}_{x}\right.$, $\left.\mathrm{Pt}_{1-x}\right)_{3} \mathrm{~B}, \mathrm{Mo}_{3} \mathrm{Al}_{2} \mathrm{C}$, and $\mathrm{BaPtSe}{ }_{3}$ all lack inversion symmetry. However, they are not strongly correlated due to the absence of $f$ or $d$ electronic configurations. ${ }^{4)}$ While the strongly correlated ones attracted attention due to the distinct experimental signatures of the ALNs and the topologically nontrivial triplet states, the others were majoritily reported as showing BCS-like conventional features. These "other s" can

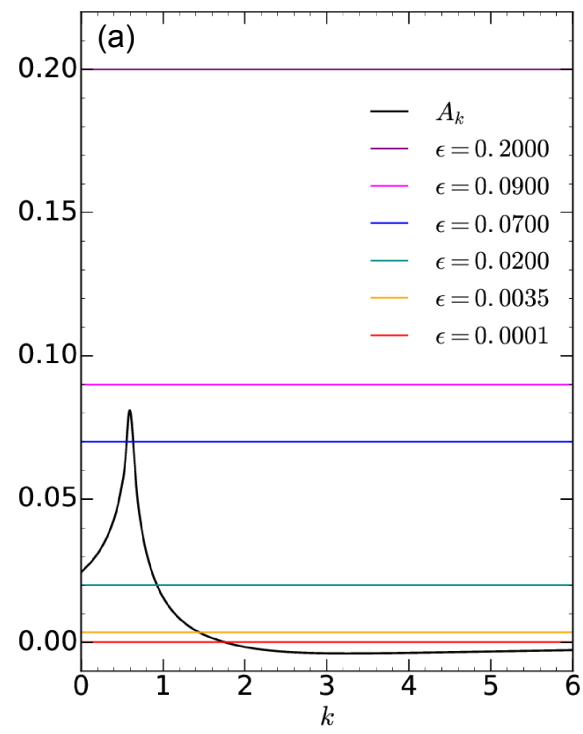

(b)

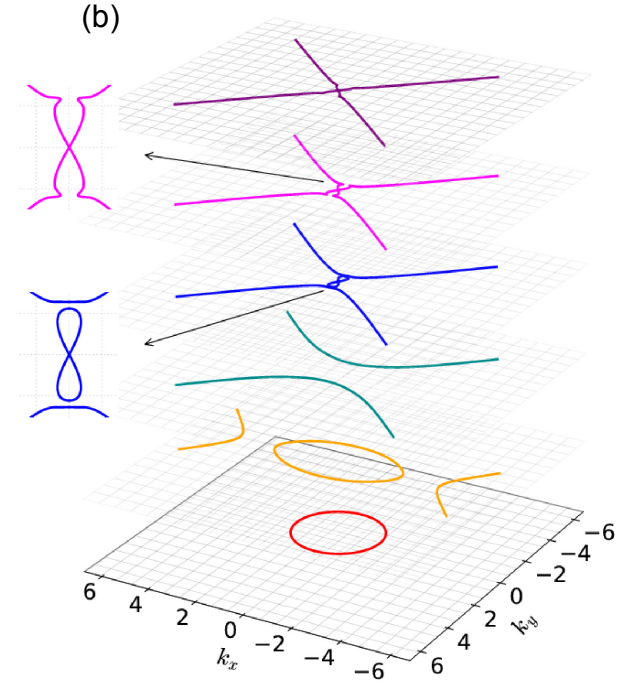

Fig. 1. (Color online) The transmutation of the nodes of $\Delta_{\mathbf{k}}^{\lambda}$ in Eq. (1) for a fixed $\lambda$ and for $A_{k}^{\lambda}$ as shown in (a). Different values of $B_{k}=\epsilon$ from an SA (upper most for $\epsilon=0.2$ ) to the WA case (lower most for $\epsilon=10^{-3}$ ) are indicated on the vertical scale in (a) and the corresponding nodes of $\Delta_{\mathbf{k}}^{\lambda}=0$ are shown in (b) with the same color coding.

have pairing potentials extending well beyond the Fermi surface and it is quite likely that they are also unconventional (non-BCS) with strongly mixed singlet and triplet states including RLNs. This important point drives the main motivation in this work. We believe that the results here are relevant to some experiments where the effect of the lattice symmetry is weak (see Sect. 3.2). With the IS broken, the relevant electronic symmetry under WA conditions is approximately $C_{\infty v}$ illustrated by the lowest ring in the Fig. 1(b). In this case, the $m=0$ component dominates with $\left|\psi_{\mathbf{k}}\right| \simeq \psi_{k}^{(0)}$ and $\left|\mathbf{d}_{\mathbf{k}}\right| \simeq F_{k}^{(0)}$ and we simply drop the (0) index.

In this section, we developed a motivation for the study of the WA regime in NCSs. In the rest of the manuscript we will focus on the topological and the thermodynamic properties in this regime. In Sect. 2 we outline the $C_{\infty v}$ symmetric model in two dimensions. Section 3 is devoted to the properties of the RLNs. Their topological properties are investigated in Sect. 3.1 and shown that the relevant class is $Z_{2}$. Section 3.2 is devoted to the low temperature thermodynamic analysis 
using mainly the energy density of states (DOS) and the specific heat. The motivation here is derived from recent experiments that in some strongly IS broken NCSs the thermodynamic data shows full isotropic gap with BCS-like exponential suppression in low temperatures seemingly pointing at the $s$-wave pairing. Arguments are raised in this section demonstrating that the thermodynamical analysis in such cases can be highly misguiding. We provide a resolution for these conflicts. Section 3.3 is devoted to the scattering at the WA N-NCS junctions. We examine the Andreev reflection spectroscopy (ARS) and show that ARS provides a suitable method to capture the distinct signatures of the WA systems.

\section{The Model}

We start with a two dimensional, $C_{\infty v}$ symmetric NCS respecting TRS. A general pairing interaction generates the singlet and the triplet components of the pair potential under a SOC. A crucial aspect is that, it is a continuum model which is maximally isotropic and no lattice point group symmetry is assumed. The Hamiltonian in the electronic Nambu-spinor basis $\Psi_{\mathbf{k}}^{\dagger}=\left(\hat{e}_{\mathbf{k} \uparrow}^{\dagger} \hat{e}_{\mathbf{k} \downarrow}^{\dagger} \hat{e}_{-\mathbf{k} \uparrow} \hat{e}_{-\mathbf{k} \downarrow}\right)$ is given by

$$
\mathcal{H}=\sum_{\mathbf{k}} \Psi_{\mathbf{k}}^{\dagger} \mathcal{H}_{\mathbf{k}} \Psi_{\mathbf{k}}=\mathcal{H}_{0}+\mathcal{H}_{\mathrm{soc}}+\mathcal{H}_{\Delta}
$$

where

$$
\mathcal{H}_{\mathbf{k}}=\left(\begin{array}{cc}
\mathcal{H}_{\mathbf{k}}^{0} & \Delta_{\mathbf{k}} \\
\Delta_{\mathbf{k}}^{\dagger} & -\left(\mathcal{H}_{-\mathbf{k}}^{0}\right)^{\mathrm{T}}
\end{array}\right)
$$

is the $4 \times 4$ mean field Hamiltonian with

$$
\mathcal{H}_{\mathbf{k}}^{0}=\xi_{\mathbf{k}} \sigma_{0}-\mathbf{G}_{\mathbf{k}} \cdot \sigma
$$

describing the kinetic and the SOC parts respectively and

$$
\Delta(\mathbf{k})=\left(\begin{array}{ll}
\Delta_{\uparrow \uparrow}(\mathbf{k}) & \Delta_{\uparrow \downarrow}(\mathbf{k}) \\
\Delta_{\downarrow \uparrow}(\mathbf{k}) & \Delta_{\downarrow \downarrow}(\mathbf{k})
\end{array}\right)=i\left(\psi_{\mathbf{k}}+\mathbf{d}_{\mathbf{k}} \cdot \sigma\right) \sigma_{y}
$$

is the pair potential. Here, $\xi_{\mathbf{k}}=\epsilon_{k}+\Sigma_{d}(\mathbf{k})$ where $\epsilon_{k}=$ $\hbar^{2} k^{2} /(2 m)-\mu, m$ is the band mass, $\mu$ is the chemical potential and $\Sigma_{d}(\mathbf{k})$ is the diagonal spin component of the self-energy. Due to the SOC, the off-diagonal contributions can generally arise in the self energy which can be effectively added in the SOC term as $\mathbf{G}_{\mathbf{k}} \rightarrow \mathbf{G}_{\mathbf{k}}+\Sigma_{o d}(\mathbf{k})$. In the Hartree-Fock mean field approach here, the self energy contributions are ignored. The elements of the OP matrix in Eq. (5) are given by

$$
\Delta_{\nu \nu^{\prime}}(\mathbf{k})=-\frac{1}{A} \sum_{\mathbf{q}} \mathcal{V}(\mathbf{q})\left\langle\hat{e}_{\mathbf{k}+\mathbf{q}, \nu}^{\dagger} \hat{e}_{-\mathbf{k}-\mathbf{q}, \nu^{\prime}}^{\dagger}\right\rangle,
$$

where $\mathcal{V}(\mathbf{q})$ is the pairing interaction and $A$ is the sample area. Here, $\Delta_{\uparrow \uparrow}(\mathbf{k})=-\Delta_{\downarrow \downarrow}^{*}(-\mathbf{k})$ by the TRS and $\Delta_{\uparrow \uparrow}(\mathbf{k})=$ $F_{\mathbf{k}} e^{-i(\phi+\pi / 2)}$, where $F_{\mathbf{k}}$ is real and even, is locked to the phase of the SOC by the unitarity of the diagonalization. These results are exact within the $C_{\infty v}$ approximation. The excitation spectrum of the Hamiltonian in Eq. (2) is given by

$$
\begin{aligned}
E_{\mathbf{k}}^{\lambda}= & {\left[\xi_{\mathbf{k}}^{2}+\left|G_{\mathbf{k}}\right|^{2}+\psi_{\mathbf{k}}^{2}+F_{\mathbf{k}}^{2}+d_{z, \mathbf{k}}^{2}\right.} \\
& \left.+2 \lambda \sqrt{\left(\xi_{\mathbf{k}}\left|G_{\mathbf{k}}\right|-\psi_{\mathbf{k}} F_{\mathbf{k}}\right)^{2}+d_{z, \mathbf{k}}^{2}\left(\left|G_{\mathbf{k}}\right|^{2}+\psi_{\mathbf{k}}^{2}\right)}\right]^{1 / 2} .
\end{aligned}
$$

The solution of the general NCS model described by Eqs. (2)-(6) requires the fully self consistent calculation of the four order parameters $\left(\psi_{\mathbf{k}}, \mathbf{d}_{\mathbf{k}}\right)$ under a general pairing interaction.
At this point we emphasize that, one of our motivations in studying the WA conditions has something to do with the relation between the $d_{\mathbf{k}}$ and $\mathbf{G}_{\mathbf{k}}$. It was shown a long time ago in Ref. 7 that $d_{\mathbf{k}} \| \mathbf{G}_{\mathbf{k}}$ yields the thermodynamically most stable configuration with the highest possible $T_{\mathrm{c}}$. It is now a common practice to employ this result in many works. It can be easily seen that, the result in Ref. 7 becomes exact in the isotropic limit studied here, and satisfied independently from the temperature and the coupling strengths. If the pairing interaction $\mathcal{V}(\mathbf{q})$ is spin independent, then $\mathcal{V}(\mathbf{q})=\mathcal{V}(q)$ where $q=|\mathbf{q}|$. The physical observables (and particularly the energy spectrum) become independent of the SOC phase $\phi$ which can be defined as a $U(1)$ gauge invariance in the particle-hole sector. An immediate consequence of this is that $d_{z, k}=0$ and it takes a few steps to show that $d_{\mathbf{k}} \| \mathbf{G}_{\mathbf{k}} \cdot{ }^{17)}$ Equation (7) can then be expressed in a generalized BCS form as ${ }^{18)}$

$$
E_{k}^{\lambda}=\sqrt{\left(\tilde{\xi}_{k}^{\lambda}\right)^{2}+\left(\tilde{\Delta}_{k}^{\lambda}\right)^{2}},
$$

where $\lambda= \pm$ is the branch index of the broken IS and

$$
\tilde{\xi}_{k}^{\lambda}=\epsilon_{k}+\lambda \gamma_{k}\left|G_{\mathbf{k}}\right| \quad \text { and } \tilde{\Delta}_{k}^{\lambda}=\left(\psi_{k}-\lambda \gamma_{k} F_{k}\right)
$$

are the single particle energy and the momentum dependent pair potential respectively with

$$
\gamma_{k}=\operatorname{sign}\left(\left|G_{\mathbf{k}}\right| \epsilon_{k}-F_{k} \psi_{k}\right) .
$$

The energy branches with $\lambda= \pm$ in Eq. (8) can have different Fermi surfaces with a different gap opening at the FS as $2\left|\tilde{\Delta}_{k}^{\lambda}\right|$. The bands are in mutual thermodynamic equilibrium by the presence of a single chemical potential, hence the Fermi level can occur at multiple positions in the $k$-space. Together with the nodes of $\tilde{\Delta}_{k}^{\lambda}$, this can give rise to a topological variety that we discuss in Sect. 3.1.

The mean field Hartree-Fock solutions of the mixed state OPs in Eq. (6) can be given in the symmetric form as

$$
\begin{aligned}
& \psi_{k}=-\frac{1}{A} \sum_{k^{\prime}, \lambda} \mathcal{V}_{s}\left(k, k^{\prime}\right) \frac{\tilde{\Delta}_{k^{\prime}}^{\lambda}}{4 E_{k^{\prime}}^{\lambda}}\left\{f\left(E_{k^{\prime}}^{\lambda}\right)-f\left(-E_{k^{\prime}}^{\lambda}\right)\right\}, \\
& F_{k}=\frac{1}{A} \sum_{k^{\prime}, \lambda} \mathcal{V}_{t}\left(k, k^{\prime}\right) \frac{\lambda \tilde{\Delta}_{k^{\prime}}^{\lambda}}{4 E_{k^{\prime}}^{\lambda}}\left\{f\left(E_{k^{\prime}}^{\lambda}\right)-f\left(-E_{k^{\prime}}^{\lambda}\right)\right\},
\end{aligned}
$$

where $f(x)=1 /[\exp (\beta x)+1]$ is the Fermi-Dirac factor with $\beta=\left(k_{\mathrm{B}} T\right)^{-1}$ as the inverse temperature. The singlet and the ESP-triplet OPs in Eqs. (11) and (12) are determined by the corresponding interaction channels $\mathcal{V}_{s}\left(k, k^{\prime}\right)$ and $\mathcal{V}_{t}\left(k, k^{\prime}\right)$. Specifically, $\quad \mathcal{V}_{s}\left(k, k^{\prime}\right)=\left\langle\mathcal{V}\left(\left|\mathbf{k}-\mathbf{k}^{\prime}\right|\right)\right\rangle_{a} \quad$ and $\quad \mathcal{V}_{t}\left(k, k^{\prime}\right)=$ $\left\langle\mathcal{V}\left(\left|\mathbf{k}-\mathbf{k}^{\prime}\right|\right) \cos \left(\phi-\phi^{\prime}\right)\right\rangle_{a}$ where $\langle\cdots\rangle_{a}$ is the angular average over the relative phase $\phi-\phi^{\prime}$. In consequence, a bare contact interaction, i.e., $\mathcal{V}\left(\left|\mathbf{k}-\mathbf{k}^{\prime}\right|\right)=U$ is insufficient to create pairing in the triplet channel even in the presence of a strong SOC. The term $\lambda \tilde{\Delta}_{k^{\prime}}^{\lambda} /\left(4 E_{k^{\prime}}^{\lambda}\right)$ in Eq. (12) is proportional to the difference between the two energy branches. However, a similar term in Eq. (11) represents the sum of the same contributions in $\psi_{k}$. A non-local pairing interaction and the SOC are therefore essential factors in the $k$-dependence of the OPs in the mixed state. This affects most importantly the RLN positions, the topology of the energy bands and the low temperature properties as discussed below.

\section{The Radial Line Nodes}

In general, whether point or line, the nodes can be present in: (a) the pair potential $\tilde{\Delta}_{k}^{\lambda}$ and (b) the energy spectrum $E_{k}^{\lambda}$. 
In SA NCSs the singlet and the triplet states are permitted by the crystal point group symmetries. The group theoretical approaches allowed the general classification of the ALNs and the energy band topology. A recent review ${ }^{19)}$ can be very useful for a complete summary. On the other hand, in the WA NCSs the point groups are ineffective and the ALNs are absent. Nevertheless, these materials can exhibit puzzling low temperature behaviour, ${ }^{5,20)}$ i.e., some NCSs with strong spin-orbit coupling display a fully gapped $s$-wave behaviour in thermodynamic response. In this work we show that the RLNs in the pair potential can resemble an isotropic $s$-wave superconductor. A fully gapped spectrum is present when the RLN positions are away from the Fermi surface, that is the case (a) above. More precisely, these type of nodes are topologically classified according to their position with respect to the Fermi surface. They can be most accurately identified by the ARPES, ${ }^{21)}$ Andreev reflection spectrosco$\mathrm{py}^{22)}$ or other ingenious measurements. ${ }^{23)}$

If an RLN is on the Fermi surface, there is a gappless spectrum and the case (b) occurs, i.e., the energy nodes in $E_{k}^{\lambda}$. Because of this additional Fermi surface matching condition, these nodes are physically rare, but when they occur, they dominate the low temperature thermodynamic behaviour. ${ }^{24)}$ Hence, a concise analysis of RLNs is necessary, which we do next.

\subsection{The topology of the RLNS}

In this section we demonstrate that the RLNs' topology is encoded in the position of the nodes and their nodal positions can, in principal, be externally controlled. This result is important from the future device applications, as experimental progress in this direction can lead us to the topologically controllable systems. The angular line nodes are, on the other hand, much less flexible externally due to the dominant effect of the crystal symmetries. We now carry on a topological analysis using two different methods and demonstrate that the topology is determined by the positions of the RLNs.

a) The block-diagonal Hamiltonian method: In the absence of the $d_{z, \mathbf{k}}$ type pairing, the mixed state Hamiltonian in Eq. (3) can be block-diagonalized in the SOC eigenbasis $\left(\hat{a}_{\mathbf{k}+}^{\dagger} \hat{a}_{\mathbf{k}-}^{\dagger} \hat{a}_{-\mathbf{k}+} \hat{a}_{-\mathbf{k}-}\right)$ where $\hat{a}_{\mathbf{k} \Lambda}=\frac{1}{\sqrt{2}}\left(\hat{e}_{\mathbf{k} \uparrow}+\lambda \gamma_{k} e^{i \phi} \hat{e}_{\mathbf{k} \downarrow}\right)$ with $\lambda= \pm$. Each block is described by a $2 \times 2$ matrix in the form written by $H^{\lambda}=\mathbf{h}_{\mathbf{k}}^{\lambda}$. $\boldsymbol{\Lambda}$ with $\boldsymbol{\Lambda}$ as the Pauli matrices in the SOC basis and $\mathbf{h}_{\mathbf{k}}^{\lambda}=\left(h_{x}^{\lambda}, h_{y}^{\lambda}, h_{z}^{\lambda}\right)=\left(\tilde{\Delta}_{k}^{\lambda} \cos \phi,-\tilde{\Delta}_{k}^{\lambda} \sin \phi, \tilde{\xi}_{k}^{\lambda}\right)$. One way to examine the energy band topology of RLNs is then to investigate each block-diagonal branch separately by the two-dimensional mapping $\left(k_{x}, k_{y}\right) \rightarrow \hat{n}_{\mathbf{k}}^{\lambda}$ where $\hat{n}_{\mathbf{k}}^{\lambda}=$ $\mathbf{h}_{\mathbf{k}}^{\lambda} / E_{\mathbf{k}}^{\lambda}$ is the Hamiltonian unit sphere. This two-dimensional mapping is described by the Chern number

$$
N_{1}^{\lambda}=\frac{1}{8 \pi} \int d^{2} k \epsilon_{i j} \hat{n}_{\mathbf{k}}^{\lambda} \cdot\left(\frac{\partial \hat{n}_{\mathbf{k}}^{\lambda}}{\partial k_{i}} \times \frac{\partial \hat{n}_{\mathbf{k}}^{\lambda}}{\partial k_{j}}\right),
$$

where $\epsilon_{i j}$ with $i, j=x, y$ is the antisymmetric tensor. The two branches can have independent indices given by the winding of $\mathbf{h}_{\mathbf{k}}^{ \pm}$on $S_{2}$.

The complete topological classification is made once all distinct configurations of the nodes in $\tilde{\Delta}_{k}^{ \pm}$relative to the position(s) of the Fermi level are identified. For this, we start with the kinetic term in the BCS-like form in Eq. (8) given by

$$
\tilde{\xi}_{k}^{\lambda}=\hbar^{2}\left(\gamma_{k} k-k_{1}^{\lambda}\right)\left(\gamma_{k} k-k_{2}^{\lambda}\right) /(2 m)
$$

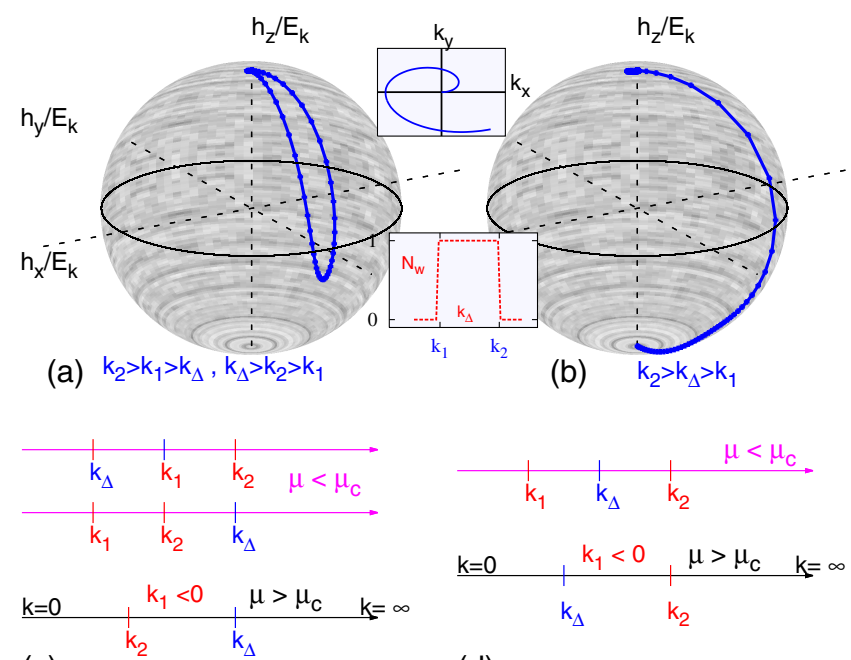

(c) Trivial-Topology

(d) Nontrivial-Topology

Fig. 2. (Color online) Nodal positions of $\tilde{\xi}_{k}^{\lambda}$ and $\Delta_{k}^{\lambda}$ when $\lambda=+$ depicted respectively as $k_{1}^{+}, k_{2}^{+}$, and $k_{\Delta}^{+}$with different topologies as indicated in (a) as trivial $N_{1}^{+}=0$, (b) as nontrivial, $N_{1}^{+}=1$. The zeros $k_{1,2}^{+}$are determined by $\mu$ and $\alpha$. The topology is illustrated on the unit sphere as (a) trivial for case (c), and (b) nontrivial for case (d).

Here $k_{1}^{\lambda}, k_{2}^{\lambda}$ are the zeros of $\tilde{\xi}_{k}^{\lambda}$. A positive $k_{j}^{\lambda}$ is a Fermi momentum on $j$ 'th Fermi surface of the corresponding branch. We assume that $k_{2}^{\lambda}>k_{1}^{\lambda}$. For the moment, we take $\gamma_{k}=1$ and discuss its effect later. The Fermi wavevectors for the + branch are

$$
\begin{aligned}
k_{2}^{+}=\frac{m}{\hbar^{2}}\left[-\alpha+\sqrt{\alpha^{2}+2 \frac{\hbar^{2}}{m}} \mu\right] & (\mu>0), \\
k_{1,2}^{+}=\frac{m}{\hbar^{2}}\left[\alpha \mp \sqrt{\alpha^{2}+2 \frac{\hbar^{2}}{m} \mu}\right] & (\mu<0) .
\end{aligned}
$$

Here $\mu=-\hbar^{2} k_{1}^{\lambda} k_{2}^{\lambda} /(2 m)$ and $\alpha=-\lambda \hbar^{2}\left(k_{1}^{\lambda}+k_{2}^{\lambda}\right) /(2 m)$ are the physical parameters which can be used to vary the $k_{1,2}^{\lambda}$. All five possibilities are shown in Fig. 2 for the + branch. The - branch is analyzed similarly.

Concentrating on the + branch, we will assume that the triplet-to-singlet ratio $\left|F_{k} / \psi_{k}\right|$ can have values smaller and larger than unity in different $k$ regions. The pair potential is then allowed to have a node, let's say at $k_{\Delta}^{+}$of the + branch, and $\Delta_{k}^{+}=\delta_{+}(k)\left(k-k_{\Delta}^{+}\right)$where $\delta_{+}(k)$ is a smooth function representing the other (irrelevant) details. At $k=k_{\Delta}^{+}$we have that $\left|\psi_{k_{\Delta}^{+}}\right|=\left|F_{k_{\Delta}^{+}}\right|$. It will also be assumed that there is only a single position where such a $k_{\Delta}^{+}$exists. In general there is nothing to prevent the number of such points to be larger than one and a complete map of such details is an additional asset to the understanding of the pairing mechanism. Particularly, a mathematical inversion of Eqs. (11) and (12) can be useful to get information about the pairing interactions by knowing the nodes of $\Delta_{k}^{\lambda}$. We nevertheless avoid this interesting idea to a later work and confine our attention to maximally one RLN at $k_{\Delta}^{\lambda}$ for each $\lambda$.

In the pure triplet state, ${ }^{25)} \psi_{\mathbf{k}}=0$ (in general realized either the TRS invariant helical $p$-wave or the TRS breaking chiral $p$-wave). In this case, the topology of the superconducting bands is decided by $\mu$ only. The Chern index in Eq. (13) is an integer yielding 


$$
N_{1}^{+}(\mu)=\left\{\begin{array}{ll}
0 & \text { for } \mu<0 \\
1 & \text { for } \mu>0
\end{array} .\right.
$$

This picture is quite similar to the $Z_{2}$ topology of the two dimensional QSHI, one dimensional polyacetylene ${ }^{26)}$ and the spinless one dimensional $p$-wave superconductor. ${ }^{27)}$

In the mixed state however, singly-parameterized characterization is not sufficient. Moreover, Eq. (13) is not integervalued. The first case is remedied by a doubly-parameterized characterization. Due to the additional spin-orbit degree of freedom, the Chern index in Eq. (13) depends on $\mu$ and $\alpha$, i.e., $N_{1}^{\lambda}(\mu, \alpha)$. The positions of $k_{1}^{\lambda}, k_{2}^{\lambda}$ relative to $k_{\Delta}^{\lambda}$ can be used to classify the topology using Eqs. (15) and (16) and the like for $\lambda=-$.

We again concentrate on the + branch. If $\mu<0$ the kinetic term can have two Fermi wavevectors $k_{1}^{+}, k_{2}^{+}>0$ given by Eq. (16), or none, i.e., $k_{1}^{+}, k_{2}^{+}<0$, whereas for $\mu>0$ there is one Fermi momentum, i.e., $k_{2}^{+}$[as given by Eq. (15)]. To begin, one can start from a trivial configuration such as $k_{1}^{+}<k_{2}^{+}<k_{\Delta}^{+}$which is then used as a reference for all other topological configurations. The mapping $\mathbf{k} \rightarrow \hat{n}_{\mathbf{k}}^{\lambda}$ is described in Figs. 2(a) and 2(b). Here, $k \rightarrow \infty$ corresponds to the north pole $(0,0,1)$ of the Hamiltonian unit sphere. The trivial and nontrivial topologies for five distinct configurations of $k_{1}^{\lambda}, k_{2}^{\lambda}$, and $k_{\Delta}^{\lambda}$ are also indicated in Figs. 2(c) and 2(d). These configurations differ in topology by the number of Fermi level crossings of the node $k_{\Delta}^{\lambda}$ where the topology is changed by every single crossing. For $k_{1}^{+}<k_{\Delta}^{+}<k_{2}^{+}, k_{\Delta}^{+}$is mapped to the south pole $(0,0,-1)$, whereas for $k_{\Delta}^{+}<k_{1}^{+}<k_{2}^{+}$, it is mapped to the north pole $(0,0,1)$.

The non-integer valued index can be remedied by considering the reduced integral range $k_{\Delta}^{\lambda} \leq k<\infty$. The results thus obtained from Eq. (13) are shown in the inset of Fig. 2.

This concludes the investigation of the block diagonal formalism. We now present another method by bringing the same Hamiltonian into a block non-diagonal form.

(b) The block-nondiagonal Hamiltonian method: A method for the topological index was proposed in Refs. 28-30 for Hamiltonians respecting "chiral symmetry" which is given by the product of the TRS and the particle hole symmetry. Both symmetries are preserved in our case here. In systems with chiral sysmmetry a new way of defining topological index can be developed by bringing the Hamiltonian in Eq. (2) into the block off-diagonal form. In our case, this is obtained by a global unitary transformation $V$ acting on $\mathcal{H}_{\mathbf{k}}$ in Eq. (3) as $^{28-30)}$

$$
V \mathcal{H}_{\mathbf{k}} V^{\dagger}=\left(\begin{array}{cc}
0 & D_{\mathbf{k}} \\
D_{\mathbf{k}}^{\dagger} & 0
\end{array}\right), \quad V=\frac{1}{\sqrt{2}}\left(\begin{array}{cc}
\sigma_{0} & -\sigma_{2} \\
i \sigma_{2} & i \sigma_{0}
\end{array}\right),
$$

where $D_{\mathbf{k}}=C_{k}\left[\cos \left(\phi_{k}\right) \sigma_{z}+i \sin \left(\phi_{k}\right) \sigma_{0}\right]-i B_{k} \sigma_{2}$ with $C_{k}=$ $\left|\mathbf{G}_{\mathbf{k}}\right|-i F_{k}$ and $B_{k}=\xi_{k}+i \psi_{k}$. Similarly to Eq. (13), here $D_{\mathbf{k}}$ is well defined only in those $\mathbf{k}$ points where the energy spectrum Eq. (8) is nonvanishing, i.e., when the gap is full. In Refs. 28-31 a momentum-dependent (contrary to global) topological index is defined as

$$
N_{2}\left(k_{\perp}\right)=\frac{1}{2 \pi} \Im m\left\{\int_{-\infty}^{\infty} d k_{\|} \partial_{k_{\|}} \ln \operatorname{det}\left(\tilde{D}_{\mathbf{k}}\right)\right\},
$$

where $k_{\|}$and $k_{\perp}$ are coordinates fully parametrizing the k-plane. Here we transformed $D_{\mathbf{k}} \rightarrow \tilde{D}_{\mathbf{k}}$ as $\operatorname{det}\left(\tilde{D}_{\mathbf{k}}\right)=$ $\operatorname{det}\left(D_{\mathbf{k}}\right) /\left|\operatorname{det}\left(D_{\mathbf{k}}\right)\right|$.
In the context of this work, $N_{2}$ is, desirably, a global index, due to the $\phi$-independence of $\operatorname{det}\left(\tilde{D}_{\mathbf{k}}\right)$. For the same reason, $k_{\|}$integral can be split into a pair of equivalent radial integrals, i.e., $k_{\|}=k$ at $\phi=0$ and $\pi$. The Eq. (19) can then be turned into

$$
N_{2}=\frac{1}{\pi} \Im m\left\{\int_{0}^{\infty} d k \partial_{k} \ln \operatorname{det}\left(\tilde{D}_{\mathbf{k}}\right)\right\} .
$$

The Eq. (20) can now be shown to be connected with $N_{1}^{\lambda}$ in Eq. (13), i.e., $N_{2}=N_{1}^{+}+N_{1}^{-}$.

For this, we use $D_{\mathbf{k}}, C_{k}$, and $B_{k}$ as defined below Eq. (18) to find that $\operatorname{det}\left(D_{\mathbf{k}}\right)=\left(\tilde{\xi}_{k}^{+}+i \tilde{\Delta}_{k}^{+}\right)\left(\tilde{\xi}_{k}^{-}+i \tilde{\Delta}_{k}^{-}\right)$. Therefore the Eq. (20) is

$$
N_{2}=\frac{1}{\pi} \sum_{\lambda} \int_{0}^{\infty} d k \partial_{k}\left[\arg \left(\tilde{\xi}_{k}^{\lambda}+i \tilde{\Delta}_{k}^{\lambda}\right)\right] .
$$

Since $\arg \left(\tilde{\xi}_{k}^{\lambda}+i \tilde{\Delta}_{k}^{\lambda}\right)$ is the polar angle $\theta^{\lambda}=\tan ^{-1} \tilde{\Delta}_{k}^{\lambda} / \tilde{\xi}_{k}^{\lambda}$ of the Hamiltonian unit vector $n^{\lambda}(\theta, \phi)=\left(\tilde{\Delta}_{k}^{\lambda} \cos \phi\right.$, $\left.\tilde{\Delta}_{k}^{\lambda} \sin \phi, \tilde{\xi}_{k}^{\lambda}\right)$, the Eq. (21) is identical with the winding of the polar angle on the unit circle at a fixed longitude $\phi^{*}$, i.e., $N_{2}=\sum_{\lambda} \int d \theta^{\lambda} / \pi$. There is therefore a one-to-one correspondence between Eqs. (13) and (21) [hence Eq. (20)].

In order to obtain an integer index from Eq. (19), the consideration of the reduced range is the simplest. Another alternative technique was also suggested. Assume that the pair potential is sufficiently weak near the Fermi surface. One can use the positions of the multiple sectors of the Fermi surface and linearly expand $\tilde{\xi}_{k}^{\lambda}$ and $\tilde{\Delta}_{k}^{\lambda}$ around the $i$ 'th Fermi surface. The expectation is that a continuous deformation assumed in the linear expansion does not change the topology, hence a discrete index is expected. It was shown in Refs. 28-31 that Eq. (19) can then be written as

$$
\begin{aligned}
N_{2}= & -\frac{1}{2} \sum_{k_{i}} \operatorname{sign}\left[\left.\partial_{k}\left(\tilde{\xi}_{k}^{+} \tilde{\xi}_{k}^{-}\right)\right|_{k=k_{i}}\right] \\
& \times \operatorname{sign}\left[\left.\left(\tilde{\Delta}_{k}^{+} \tilde{\xi}_{k}^{-}+\tilde{\Delta}_{k}^{-} \tilde{\xi}_{k}^{+}\right)\right|_{k=k_{i}}\right],
\end{aligned}
$$

where point(s) $k_{i}^{\lambda}$ are the Fermi momenta given by $\left.\tilde{\xi}_{k}^{\lambda}\right|_{k_{i}^{\lambda}}=0$. This can be written as a sum of separate branches as

$$
N_{2}=-\frac{1}{2} \sum_{\lambda} \sum_{\tilde{\xi}_{k_{i}}^{\lambda}=0} \operatorname{sign}\left[\left.\partial_{k} \tilde{\xi}_{k}^{\lambda}\right|_{k=k_{i}}\right] \operatorname{sign}\left[\left.\tilde{\Delta}_{k}^{\lambda}\right|_{k=k_{i}}\right] \text {. }
$$

The $N_{2}^{\lambda}$ calculated from Eq. (23) yields the same result as $N_{1}^{\lambda}$ using the reduced integration range (see Fig. 2). With the equivalence of both methods in parts Figs. 2(a) and 2(b), we revisit Figs. 2(c) and 2(d) for a summary. The topological indices defined in this section are undefined when the spectral gap closes. The topology of the RLNs in the full gap configuration requires that $k_{\Delta}^{\lambda}$ is not on the Fermi surface, i.e., $k_{\Delta}^{\lambda} \neq\left(k_{1}^{\lambda}, k_{2}^{\lambda}\right)$. The topology can then be classified by the $Z_{2}$ index according to the position of the RLN in the pair potential with respect to the Fermi surface. This requires that the configurations where $k_{\Delta}^{\lambda}=k_{1}^{\lambda}$ or $k_{\Delta}^{\lambda}=k_{2}^{\lambda}$ are topologically undefined. Since these specific configurations are where the energy gap closes, we have the important result that, an RLN in the energy spectrum occurs at the boundary of two distinct topological regions. This concludes our discussion on the topology of the RLNs. We now investigate the influence of the RLNs on the thermodynamic observables where the results of Sect. 3.1 will also be used. 


\subsection{Thermodynamic signatures}

In fully gapped NCSs, specific heat, penetration depth and other thermodynamic observables display exponential suppression in temperature in sufficiently low temperatures. For this reason, it is difficult in thermodynamic experiments to separate the unconventional pairing in these systems from the fully gapped trivial $s$-wave superconductors.

On the other hand, the gapless superconductors - mostly studied in the context of ALNs in the anisotropic regimecan be easily identified in thermodynamic measurements with their distinct scaling behaviour near vanishing excitation energies. In this case, the exponential suppression in temperature is replaced by a clean power law depending on the nodal dimensions. It is known that in two dimensional systems, the point nodes can yield in the specific heat a $T^{3}$, whereas the ALNs yield a $T^{2}$ dependence. ${ }^{4)}$

It is our goal in this section to show that, the WA systems where ALNs are not present, behave thermodynamically like the ordinary $s$-wave superconductors. This is so even in the presence of strongly mixed singlet-triplet components with RLNs present in the pair potential. In order to study the thermodynamics of these systems, we start with the energy DOS of the branch $\lambda$,

$$
\rho_{\lambda}(E)=\int \frac{d \mathbf{k}}{(2 \pi)^{2}} \delta\left(E-E_{\mathbf{k}}^{\lambda}\right)
$$

and examine its behaviour in the context of Sect. 3.1. We consider $E_{k}^{\lambda}$ in the context of Eqs. (8) and (14) also allowing the pair potential to have a simple RLN at $k_{\Delta}^{\lambda}$, i.e., $\Delta_{k}^{\lambda} \simeq$ $b_{\lambda}\left(k-k_{\Delta}^{\lambda}\right)$. If we concentrate on the region $k \simeq k_{2}^{\lambda}$ for a fixed $\lambda$ and $\mu>0$, then $\tilde{\xi}_{k}^{\lambda} \simeq a_{\lambda}\left(k-k_{2}^{\lambda}\right)$. Here $a_{\lambda}$ and $b_{\lambda}$ are some coefficients. We find that

$$
\rho_{\lambda}(E)=\left.\frac{1}{2 \pi} \frac{E}{a_{\lambda}^{2}\left(1-k_{2}^{\lambda} / k\right)+b_{\lambda}^{2}\left(1-k_{\Delta}^{\lambda} / k\right)}\right|_{k=k_{\lambda}(E)}
$$

and $k_{\lambda}(E)$ is where $E_{k}^{\lambda}=E$. Equation (25) indicates that, for large energies $\rho(E) \sim E$. The small energy limit of DOS depends on whether a zero energy mode at a finite $k$ is supported in the spectrum. For the zero energy mode $k_{2}^{\lambda}=$ $k_{\Delta}^{\lambda}$ must be physically realized, i.e., the node in the pair potential must occur at the Fermi level. In this case, Eq. (24) implies that in the vicinity of the zero mode $\rho_{\lambda}(E)=$ $k_{E}^{\lambda} /\left(2 \pi \sqrt{a_{\lambda}^{2}+b_{\lambda}^{2}}\right)$, i.e., a constant. On the other hand, if $k_{2}^{\lambda} \neq k_{\Delta}^{\lambda}$, there is a gap in the spectrum for $E<E_{\min }^{\lambda}=$ $a_{\lambda} b_{\lambda}\left|k_{2}^{\lambda}-k_{\Delta}^{\lambda}\right| / \sqrt{a_{\lambda}^{2}+b_{\lambda}^{2}}$ with a divergent DOS at the gap edge. The DOS for this $\mu>0$ case is summarized in Fig. 3(a). Before commenting on this case, we examine the DOS for $\mu<0$. Here, there are two Fermi surface positions or none. Let us assume that there can be one RLN of the pair potential at $k_{\Delta}^{\lambda}$ for each branch $\lambda$. In this case, there can be two, one or zero number of energy nodes and the picture obtained for the $\mu>0$ case in the DOS is repeated here according to the number of energy nodes. Finally, the DOS for $\mu<0$ is shown in Fig. 3(b).

In Figs. 3(a) and 3(b), the behaviour of the DOS in the fully gapped regimes is unseparable from a conventional $s$ wave superconductor. Different behaviour from the conventional superconductor appears when the node is located on the Fermi surface. This corresponds to an RLN in the energy spectrum and in contrast to the ALNs where $\rho(E) \sim E^{\nu}$ with $\nu$ being an integer exponent depending on dimensionality, ${ }^{4)}$ here the DOS acquires a constant value. A comparison with the previous section shows that, the point where the discontinuous jump occurs in $\rho(0)$ is a boundary between topologically two distinct regimes. The experimental observation of this discontinuity should be considered as a significant evidence about the presence of RLNs and, any thermodynamic quantity based on $\rho(E)$ is expected to have this signature. For instance the specific heat given by

$$
C_{V}(T)=\sum_{\lambda} \int d E \rho_{\lambda}(E) E \frac{f(E)}{d T},
$$

where $f(E)$ was define before, displays a sharp transition from the exponential suppression to the linear dependence as shown in Figs. 3(c) and 3(d). The temperature dependence of the $C_{V}$ in an NCS with RLNs is therefore very similar to that of the $s$-wave BCS superconductor. This is a crucial information which may be useful in resolving some of the experimental controversies. Indeed, recently a number of thermodynamic experiments were reported on NCSs with strong IS breaking ${ }^{20)}$ and the list is rapidly extending. ${ }^{32)}$ In these works, the thermodynamic data is similar to Figs. 3(c) and 3(d) and the opinion of those authors is in favour of the conventional $s$-wave BCS superconductivity. On the other hand, other evidences were also emphasized therein pointing at the unconventional pairing.

Our results in this section can demonstrate that a fully gapped superconductor with an RLN located off the Fermi surface can display thermodynamic data like ordinary $s$-wave superconductors at the same time being topologically unconventional in a strongly mixed state.

An important side remark is that, if $\gamma_{k}$ changes sign between the two Fermi wavevectors $k_{1}$ and $k_{2}$, then both gaps $\Delta_{k}^{ \pm}$are allowed to have RLNs. This case is interesting but certainly a very rare circumstance. Its experimental identification may be difficult to reveal in thermodynamic measurements, but it may be possible by ARS which we discuss next.

\subsection{The Andreev reflection spectroscopy with WA NCSs}

The arguments raised above show that the thermodynamic data can be misleading in understanding the OP symmetry. We furthermore demonstrated that, the RLNs offer an explanation to these controversies and they are most likely present in WA systems.

We can differentiate the SA conditions from the WA ones, for instance as shown in the top and the bottom slices of Fig. 1(b), by using probes that can control the energy and the momentum vector at the single particle level. The Andreev conductance (AC) measurements have been useful experimental tools for obtaining information about the pairing symmetry of the s, $\mathrm{d}$ and chiral $p$-wave superconductors. ${ }^{4,33)}$ In this section, we demonstrate that the $\mathrm{AC}$ can be also an efficient probe for the WA ones.

We consider the junction of a normal metal $(\mathrm{N})$ with a WA NCS in the $x-y$ plane and calculate the AC at an N-NCS interface where $x<0$ is the $\mathrm{N}$ side without SOC and $x>0$ is the NCS with SOC. We assume ideal conditions with no interface potential which allows us to discard the normal reflection. ${ }^{34)}$

Initially, an electron, spin-polarized in the $z$-direction, is sent normal to the $\mathrm{N}-\mathrm{NCS}$ interface from the $\mathrm{N}$ side at the wavevector $K_{e}=\sqrt{2 m\left(E+\mu_{\mathrm{N}}\right) / \hbar^{2}}$ where $E$ is the energy of 

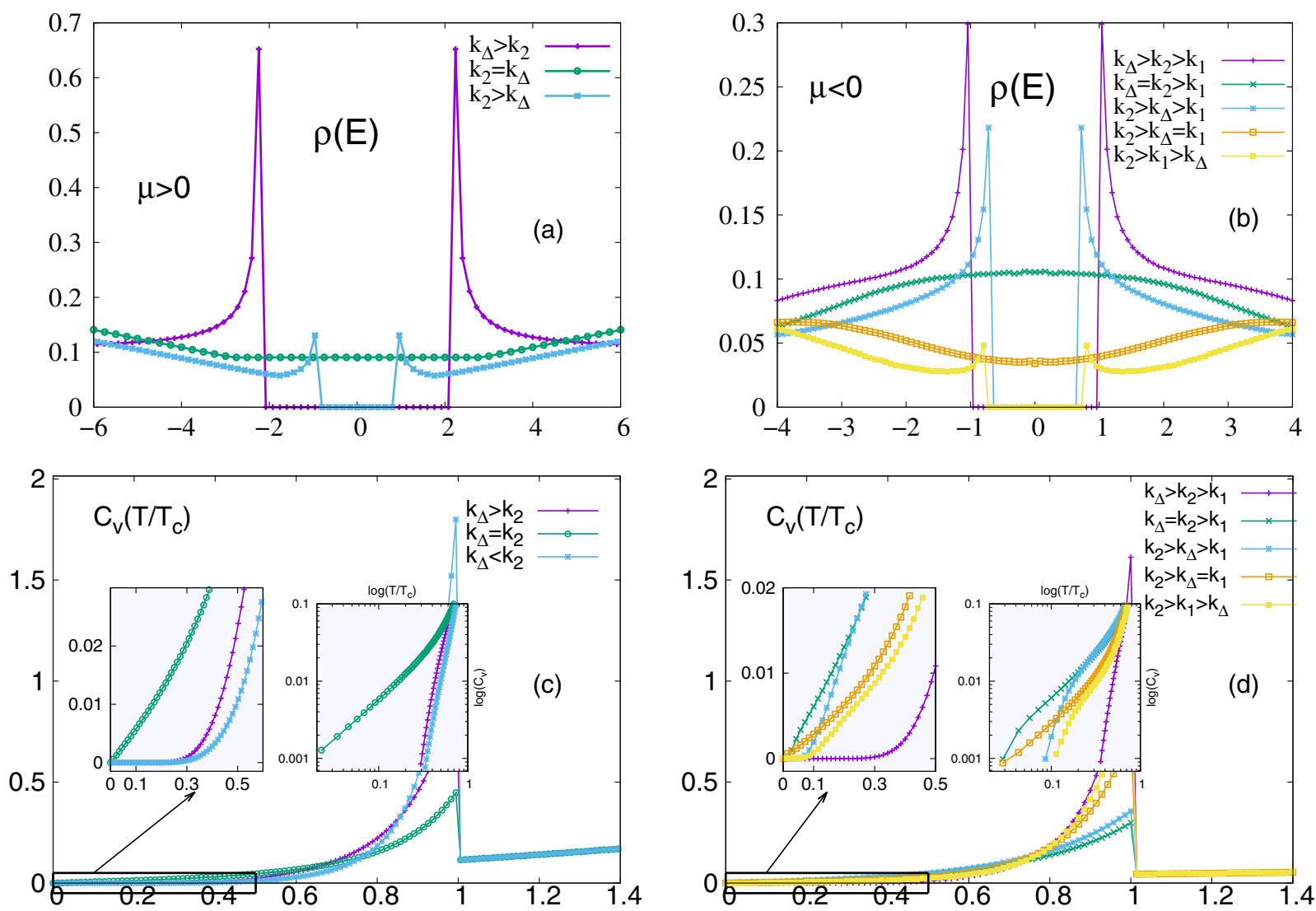

Fig. 3. (Color online) The effect of the Fermi level crossing of the node $k_{\Delta}$ in the pairing potential for $\mu>0$ in (a) the DOS $\rho(E)$ and (b) the $C_{V}$ corresponding to the cases $k_{\Delta}<k_{2}, k_{\Delta}=k_{2}$, and $k_{\Delta}>k_{2}$. The effect of the Fermi level crossing of the energy gap node $k_{\Delta}$ for $\mu<0$ on the (a) $\rho$ (E) and (b) $C_{V}$ corresponding to five different positions of $k_{\Delta}$ color coded in (b), as also indicated in Figs. 2(a) and 2(b). The insets magnify the low $E$ and low $T$ region of $\rho(E)$ and $C_{V}$ which are linear for $k_{\Delta}=k_{1}$ and $k_{\Delta}=k_{2}$.

the incident electron and $\mu_{\mathrm{N}}=\hbar^{2} k_{\mathrm{F}}^{2} /(2 m)$ is the chemical potential in the $\mathrm{N}$ region with $k_{\mathrm{F}}$ being the Fermi wavevector in the $\mathrm{N}$ side. We perform our calculations for the zero voltage bias, i.e., $\mu_{\mathrm{N}}=\mu_{\mathrm{NCS}}$. The corresponding wavefunctions are ${ }^{35)}$

$$
\begin{aligned}
\Psi_{\mathrm{N}}(x)= & \left\{e^{i K_{e} x}(1,0,0,0)^{\mathrm{T}}+a e^{i K_{h} x}(0,0,1,0)^{\mathrm{T}}\right. \\
& +b e^{i K_{h} x}(0,0,0,1)^{\mathrm{T}}+c e^{-i K_{e} x}(1,0,0,0)^{\mathrm{T}} \\
& \left.+d e^{-i K_{e} x}(0,1,0,0)^{\mathrm{T}}\right\}, \\
\Psi_{\mathrm{S}}(x)= & \left\{c_{1} e^{i q_{1}^{+} x}\left(u_{1}, \eta u_{1}, \eta^{*} v_{1}, v_{1}\right)^{\mathrm{T}}\right. \\
& +c_{2} e^{i q_{2}^{+} x}\left(-\eta^{*} u_{2}, u_{2}, v_{2},-\eta^{*} v_{2}\right)^{\mathrm{T}} \\
& +c_{3} e^{-i q_{1}^{-} x}\left(-\eta^{*} v_{1}, v_{1}, u_{1},-\eta^{*} u_{1}\right)^{\mathrm{T}} \\
& \left.+c_{4} e^{-i q_{2}^{-} x}\left(v_{2}, \eta v_{2}, \eta u_{2}, u_{2}\right)^{\mathrm{T}}\right\},
\end{aligned}
$$

where $\eta=e^{i \phi}$ evaluated at the corresponding transmitted wavevector $q_{\left(\begin{array}{l}1 \\ 2\end{array}\right)}^{ \pm}$in Eq. (27), and for $|E|>\left|\Delta_{ \pm}\right|$

$$
\begin{aligned}
& u_{\left(\frac{1}{2}\right)}=\frac{1}{2} \sqrt{\left(1+\sqrt{1-\left(\frac{\Delta_{ \pm}}{E}\right)^{2}}\right)}, \\
& v_{\left(\frac{1}{2}\right)}=\frac{1}{2} \sqrt{\left(1-\sqrt{1-\left(\frac{\Delta_{ \pm}}{E}\right)^{2}}\right)},
\end{aligned}
$$

whereas analogous to the BTK theory, ${ }^{34)}$ the coefficients are complex for $|E|<\left|\Delta_{ \pm}\right|$as,

$$
u_{\left(\frac{1}{2}\right)}=\frac{1}{2} \sqrt{\left|\frac{\Delta_{ \pm}}{E}\right|} e^{i \theta_{ \pm}}, \quad \theta_{ \pm}=\tan ^{-1} \sqrt{\left|\frac{\Delta_{ \pm}}{E}\right|^{2}-1},
$$

and $v_{\left(\frac{1}{2}\right)}^{*}=u_{\left(\frac{1}{2}\right)}$. Here $a, b$ are the complex Andreev reflection amplitudes for the hole with wavevector $K_{h}=$ $\sqrt{2 m\left(-E+\mu_{\mathrm{N}}\right) / \hbar^{2}}$, and the $c, d$ are the normal reflection amplitudes. On the NCS side, $c_{1}, \ldots, c_{4}$ are the transmission amplitudes within the NCS in the \pm branches. Here $c=$ $d=0$ as the result of the absence of normal reflection as mentioned before. Hence only $a, b$ are present due to the Andreev mechanism.

The pair potential $\Delta_{k}^{\lambda}$ is an isotropic function of $k=$ $\sqrt{k_{x}^{2}+k_{y}^{2}}$ in the NCS. Due to the homogeneous boundary conditions along the $y$-direction, the $k_{y}$ is conserved across the boundary. The $\Delta_{k}^{\lambda}$ is therefore a function of $E$ of the incident probe particle and its angle of incidence $\phi_{i}$ on the $N$ side. For normal incidence, we take $\phi_{i}=0$. This being the case for the perfect isotropy, for WA NCSs the pair potential can be a weak function of the orientaton angle $\phi_{0}$ of the crystal axes relative to the interface plane in the NCS. In this case, $\Delta_{k}^{\lambda}$ can be considered as a fuzzy function of $E$ with a narrow spread given by the degree of anisotropy. In the isotropic limit, fuzziness disappears and $\Delta_{k}^{\lambda}=\Delta^{\lambda}(E)$ becomes a sharp function of $E$. Assuming this last case and for a given initial energy $E$, there are three different regimes: a) $E<\left|\Delta_{-}\right|<\left|\Delta_{+}\right|$where $\left|u_{1}^{2}\right|=\left|v_{1}^{2}\right|$ and $\left|u_{2}^{2}\right|=\left|v_{2}^{2}\right|$, b) $\left|\Delta_{-}\right|<E<\left|\Delta_{+}\right|$where $\left|u_{1}^{2}\right|=\left|v_{1}^{2}\right|$ and $\left|u_{2}^{2}\right| \neq\left|v_{2}^{2}\right|$, and c) $\left|\Delta_{-}\right|<\left|\Delta_{+}\right|<E$ where $\left|u_{1}^{2}\right| \neq\left|v_{1}^{2}\right|$ and $\left|u_{2}^{2}\right| \neq\left|v_{2}^{2}\right|$. We 

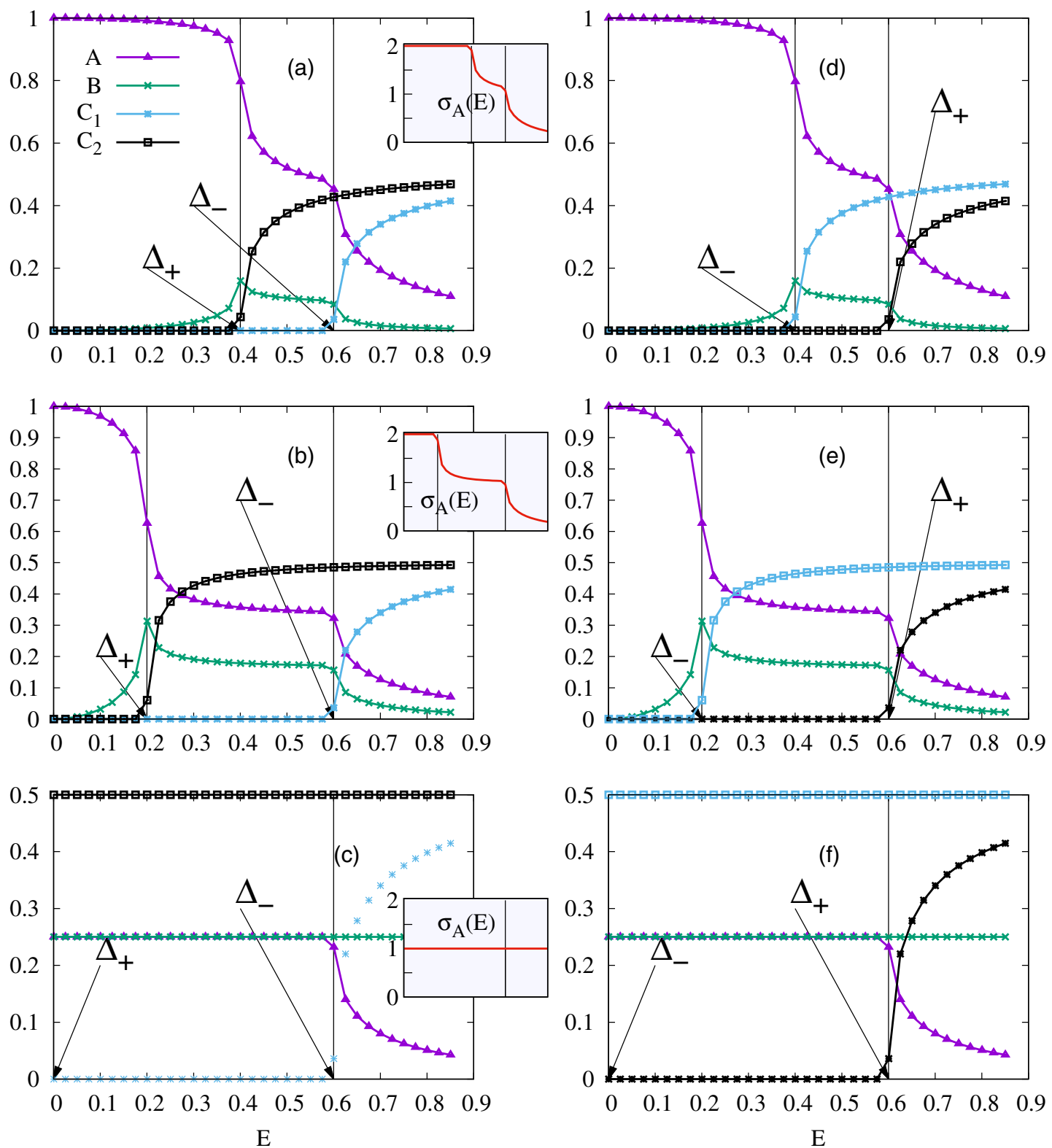

Fig. 4. (Color online) Spin resolved Andreev reflection and the transmission probabilities in an N-NCS interface are depicted with Andreev conductance $\sigma_{A}$ (in units of $2 e^{2} / h$ in the inset for each horizontal case) for three different configurations: (a) $\Delta_{-}>\Delta_{+}>0$, (b) same as (a) when $\Delta_{+}$is lowered, and (c) same as (a) when $\Delta_{+}=0$. The right columns (c, e, f) correspond to the cases $\Delta_{-} \leftrightarrow \Delta_{+}$.

assumed here that $\left|\Delta_{-}(E)\right|<\left|\Delta_{+}(E)\right|$ which may or may not be true for all energies (details are in the caption of Fig. 4). The results here are unaffected by such details.

The full solution of the coefficients in Eq. (27), requiring the application of the boundary conditions $\Psi_{\mathrm{N}}(x)=$ $\Psi_{\mathrm{S}}(x)$ and $\Psi_{\mathrm{N}}^{\prime}(x)=\Psi_{\mathrm{S}}^{\prime}(x)$ at the $x=0$ interface, has been shown in a large number of works and will not be shown here. The continuity of the current at the interface requires that

$$
1-\left(|a|^{2}+|b|^{2}\right)=S_{a, b, c}
$$

where

$$
\begin{aligned}
& S_{a}=z, \\
& S_{b}=\frac{q_{2}^{+}}{K_{e}}\left|c_{2}\right|^{2}\left(\left|u_{2}\right|^{2}-\left|v_{2}\right|^{2}\right), \\
& S_{c}=\frac{q_{1}^{+}}{K_{e}}\left|c_{1}\right|^{2}\left(\left|u_{1}\right|^{2}-\left|v_{1}\right|^{2}\right)+\frac{q_{2}^{+}}{K_{e}}\left|c_{2}\right|^{2}\left(\left|u_{2}\right|^{2}-\left|v_{2}\right|^{2}\right)
\end{aligned}
$$

with $S_{a, b, c}$ corresponding to the three cases above. The probabilities $A=|a|^{2}$ and $B=|b|^{2}$ are the Andreev reflection probabilities $^{4,35)}$ for the hole in $x<0$, whereas $C_{1}=$ $\frac{q_{1}^{+}}{K_{e}}\left|c_{1}\right|^{2}\left(\left|u_{1}\right|^{2}-\left|v_{1}\right|^{2}\right), C_{2}=\frac{q_{2}^{+}}{K_{e}}\left|c_{2}\right|^{2}\left(\left|u_{2}\right|^{2}-\left|v_{2}\right|^{2}\right)$ are for the transmission probabilities corresponding to the \pm branches. Also, $c_{3}=c_{4}=0$ due to the absence of reflection in a semiinfinite geometry in the NCS. Here we will be interested in the double step-like behaviour of the $A=|a|^{2}$ and $B=|b|^{2}$ as a result of the Eq. (31) as shown in Fig. 4.

Respecting these three energetically different regimes, we examine the $\mathrm{AC}\left(\sigma_{A}\right)$ in two distinct cases of the pair potentials: (i) when both $\tilde{\Delta}^{ \pm}$are nonzero and, (ii) when one of them is zero at a nodal position. The spin dependent Andreev reflection and the transmission coefficients are shown in the Figs. 4(a)-4(f) together with the AC $\sigma_{A}$ calculations in the insets, where the mutual positions of the $\Delta^{ \pm}$are varied in Figs. 4(a), 4(b), 4(d), 4(e) and one of the pair potentials is assumed to be at the nodal position at 

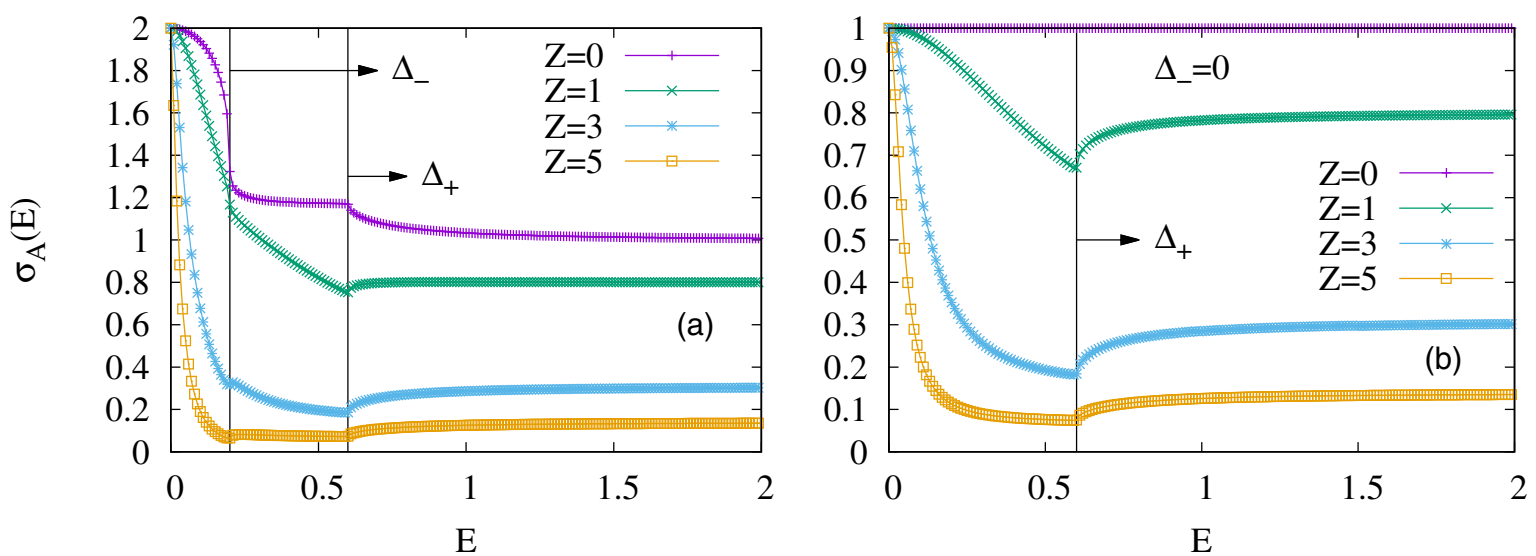

Fig. 5. (Color online) The $\sigma_{A}(E)$ (in units of $2 e^{2} / h$ ) for a semi infinite N-NCS interface including a delta-function-like interface potential $Z \delta(x)$ when (a) both pair potentials are nonzero, (b) $\Delta^{-}=0$ and $\Delta^{+} \neq 0$. The $Z$ values are given in units of an energy scale equivalent to 10 meV.

$E=0$ in Figs. 4(c) and 4(f). Let us concentrate on the inset in Fig. 4(a) where the $\sigma_{A}$ starts with a plateau at unit conductance corresponding to the configuration $E<\left|\Delta_{-}\right|<$ $\left|\Delta_{+}\right|$, i.e., case (a) defined above Eq. (30). If $E$ is between the gaps, as in case (b), the AC moves down to a second plateau. For the case (c), the $\sigma_{A}$ gradually disappears as $E$ is increased. If the interval $\left|\Delta^{+}-\Delta^{-}\right|$is changed, the curves change only quantitatively with the double-step behaviour unchanged [Figs. 4(b) and 4(e)]. We now shift to the second distinct case (ii) above, when one of the pair potentials is zero as shown in Figs. 4(c) and 4(f) corresponding to a RLN position. The $\sigma_{A}$ in the inset therein, directly starts at $1 / 2$ at low energies then going through a single plateau before gradually vanishing at high energies. If this RLN is located on the Fermi surface, this should experimentally give rise to a narrow zero bias conductance peak.

The double steps in the AC is a signature of the three regions with different Andreev reflection properties. We therefore expect that the two distinct steps should always be present where the other details such as the step length and the vertical range of the steps should be material dependent. From the theoretical point of view, double steps clearly point at the WA conditions, but in reality there can be a weak dependence on the orientation angle $\phi_{0}$ in $\Delta^{ \pm}$. This should affect the steepness of the falls in $\sigma_{A}$ between the plateaus. However, there can be a serious danger on the visibility of the double steps: these characteristic features can be completely erased in the presence of imperfections on the interface as we discuss now.

We checked the robustness of the double steps against the imperfections on the interface. In this case, we expect that an effective transmission barrier is created on the interface which can easily obscure the ideal AC profile. To understand this effect, we assumed a spin-neutral barrier potential as $Z \delta(x)$ with $Z$ describing the barrier strength and then calculated the AC. Our results, as shown in Fig. 5, confirm that, the shape of the $\mathrm{AC}$ is extremely fragile against the surface imperfections and the double step behaviour is destroyed completely. Highly clean interfaces are therefore needed to observe the ideal double step behaviour.

\section{Conclusions}

In this work, we concentrated on the topology and the thermodynamics of the WA NCSs. The RLNs can emerge in these systems, in contrast to the ALNs appearing under SA conditions. In an RLN, the pair potential vanishes in certain reciprocal space points where the singlet and the triplet couplings become locally comparable. They are pronounced in WA systems where their more anisotropic counterparts (the ALNs) are usually absent. The low temperature dynamics caused by RLNs is dintinctly different from that of the ALNs. In particular, when they are not on the Fermi surface, the RLNs can imitate an isotropic BCS like full gap behaviour in the low temperature thermodynamic measurements without giving up the unconventional pairing and the topology. This finding is crucial in that, a number of experimental results on NCSs in favour of trivial s-wave coupling may need to be reconsidered and these results may happily turn out to be nontrivial. Furthermore several compounds with broken TRS are reported to show a similar behaviour. ${ }^{36)}$ The picture presented by the RLNs may be also relevant in the TRS broken NCSs and this deserves a separate work.

The topology of the energy bands is classified by the relative position of the RLN in the pair potential with respect to the Fermi wavevector. The latter can be shifted by the chemical potential and the SOC, bringing an additional importance that the topology can be manipulated externally by electrostatic gates and the SOC strength. This can open promising research directions based on superconducting node engineering with implications in exotic device applications in topological quantum computing. Finally, the Andreev spectroscopy in N-NCS junctions is an efficient tool for probing the double gap structure of the WA NCSs where RLNs are most likely to be found experimentally.

For the RLNs to exist in a mixed singlet-triplet state, and besides the SOC, it is sufficient to have an isotopic component of the pairing interaction sufficiently attractive in the long ranges. On the other some of the isotropic NCSs in the scope of this work with large values of the universal BCS ratio and the anomalous specific heat jumps may be pointing at strong and/or long range interactions. We have not referred to a particular pairing mechanism in this manuscript. Our main results are thus expected to be valid in the strong coupling extention of our work which can be developed in the future. 
1) R. Balian and R. N. Werthamer, Phys. Rev. 131, 1553 (1963); P. W. Anderson and P. Morel, Phys. Rev. 123, 1911 (1961); A. J. Leggett, Phys. Rev. Lett. 29, 1227 (1972).

2) R. H. Heffner and M. R. Norman, Comments Condens. Matter Phys. 17, 361 (1996); H. R. Ott, H. Rudigier, Z. Fisk, and J. L. Smith, Phys. Rev. Lett. 50, 1595 (1983); F. Steglich, J. Aarts, C. D. Bredl, W. Lieke, D. Meschede, W. Franz, and H. Schafer, Phys. Rev. Lett. 43, 1892 (1979).

3) J. G. Bednorz and K. A. Müller, Z. Phys. B 64, 189 (1986); P. A. Lee, N. Nagaosa, and X.-G. Wen, Rev. Mod. Phys. 78, 17 (2006).

4) Noncentrosymmetric Superconductors, ed. E. Bauer and M. Sigrist (Springer, New York, 2012) Lecture Notes in Physics, Vol. 847; S Yip, Annu. Rev. Condens. Matter Phys. 5, 15 (2014).

5) F. Kneidinger, E. Bauer, I. Zeiringer, P. Rogl, C. Blaas-Schenner, D. Reith, and R. Podloucky, Physica C 514, 388 (2015).

6) K. Izawa, H. Takahashi, H. Yamaguchi, Y. Matsuda, M. Suzuki, T. Sasaki, T. Fukase, Y. Yoshia, R. Settai, and Y. Onuki, Phys. Rev. Lett. 86, 2653 (2001).

7) P. A. Frigeri, D. F. Agterberg, A. Koga, and M. Sigrist, Phys. Rev. Lett. 92, 097001 (2004)

8) T. Hakioğlu and M. Şahin, Phys. Rev. Lett. 98, 166405 (2007); M. A. Can and T. Hakioğlu, Phys. Rev. Lett. 103, 086404 (2009).

9) W. Liu and A. Punnoose, Phys. Rev. Lett. 114, 176402 (2015); G. M. Luke, Y. Fudamoto, K. M. Kojima, M. I. Larkin, J. Merrin, B. Nachumi, Y. J. Uemura, Y. Maeno, Z. Q. Mao, Y. Mori, H. Nakamura, and M. Sigrist, Nature 394, 558 (1998).

10) Theory of Unconventional Superconductors, ed. D. Manske (Springer, New York, 2004); Unconventional Superconductors, ed. G. Goll (Springer, New York, 2006); Vortices in Unconventional Superconductors and Superfluids, ed. R. P. Huebener, N. Schopohl, and G. E. Volovik (Springer, New York, 2002).

11) H. Q. Yuan, D. F. Agterberg, N. Hayashi, P. Badica, D. Vandervelde, K. Togano, M. Sigrist, and M. B. Salamon, Phys. Rev. Lett. 97, 017006 (2006); K. Ishida, H. Mukuda, Y. Kitaoka, K. Asayama, Z. Q. Mao, Y. Mori, and Y. Maeno, Nature 396, 658 (1998); Y. Maeno, H. Hashimoto, K. Yoshida, S. Nishizaki, T. Fujita, J. G. Bednorz, and F. Lichtenberg, Nature 372, 532 (1994); A. P. Mackenzie and Y. Maeno, Rev. Mod. Phys. 75, 657 (2003).

12) K. Izawa, Y. Kasahara, Y. Matsuda, K. Behnia, T. Yasuda, R. Settai, and Y. Onuki, Phys. Rev. Lett. 94, 197002 (2005); I. Bonalde, W. Brämer-Escamilla, and E. Bauer, Phys. Rev. Lett. 94, 207002 (2005); J. Chen, M. B. Salamon, S. Akutagawa, J. Akimitsu, J. Singleton, J. L. Zhang, L. Jiao, and H. Q. Yuan, Phys. Rev. B 83, 144529 (2011).

13) A New Method in the Theory of Superconductivity, ed. N. N Bogoliubov, V. V. Tolmachev, and D. V. Shirkov (Consultants Bureau, New York, 1959); Superconductivity of Metals and Alloys, ed P. G. de Gennes (W. A. Benjamin, New York, 1966); P. W. Anderson and P. Morel, Phys. Rev. B 123, 1911 (1961).

14) M. Sigrist and K. Ueda, Rev. Mod. Phys. 63, 239 (1991); A. B. Vorontsov, I. Vekhter, and M. Eschrig, J. Phys. Soc. Jpn. 77 [Suppl. A], 165 (2008).

15) T. Hakioğlu and M. Günay, Physica C 528, 48 (2016).

16) T.-R. Chang, P.-J. Chen, G. Bian, S.-M. Huang, H. Zheng, T. Neupert, R. Sankar, S.-Y. Xu, I. Belopolski, G. Chang, B. Wang, F. Chou, A. Bansil, H.-T. Jeng, H. Lin, and M. Z. Hasan, Phys. Rev. 93, 245130 (2016); S. Sasaki, Z. Ren, A. A. Taskin, K. Segawa, L. Fu, and Y. Ando, Phys. Rev. Lett. 109, 217004 (2012); A. S. Alexandrov, Phys. Rev. B 77, 094502 (2008).

17) The Eq. (7) and $\left(\psi_{\mathbf{k}}, F_{\mathbf{k}}, d_{z \mathbf{k}}\right)$ are independent of $\phi$, i.e., given by $\left(\psi_{k}, F_{k}, d_{z k}\right)$. This result agrees with the even parity of $\psi_{\mathbf{k}}, F_{\mathbf{k}}$, whereas it does contradict with the odd parity of $d_{z \mathbf{k}}$ under inversion. Hence it must be that $d_{z \mathbf{k}}=d_{z k}=0$. This result, together with the phase-locked $\Delta_{\uparrow \uparrow}(\mathbf{k})$ below Eq. (6) implies $\mathbf{d}_{\mathbf{k}} \| \mathbf{G}_{\mathbf{k}}$.

18) The original spectrum with the full in Eq. (7) is not expressible in a generalized BCS form similar to Eq. (8).
19) M. Smidman, M. B. Salamon, H. Q. Yuan, and D. F. Agterberg, Rep. Prog. Phys. 80, 036501 (2017).

20) G. Eguchi, H. Wadati, T. Sugiyama, E. Ikenaga, S. Yonezawa, and Y. Maeno, Phys. Rev. B 86, 184510 (2012); J. Chen, L. Jiao, J. L. Zhang, Y. Chen, L. Yang, M. Nicklas, F. Steglich, and H. Q. Yuan, Phys. Rev. B 88, 144510 (2013); S. Y. Zhou, X. L. Li, B. Y. Pan, X. Qiu, J. Pan, X. C. Hong, Z. Zhang, A. F. Fang, N. L. Wang, and S. Y. Li, Europhys. Lett. 104, 27010 (2013); C. Cirillo, R. Fittipaldi, M. Smidman, G. Carapella, C. Attanasio, A. Vecchione, R. P. Singh, M. R. Lees, G. Balakrishnan, and M. Cuoco, Phys. Rev. B 91, 134508 (2015).

21) C. N. Veenstra, Z.-H. Zhu, M. Raichle, B. M. Ludbrook, A. Nicolaou, B. Slomski, G. Landolt, S. Kittaka, Y. Maeno, J. H. Dil, I. S. Elfimov, M. W. Haverkort, and A. Damascelli, Phys. Rev. Lett. 112, 127002 (2014).

22) D. Daghero and R. S. Gonnelli, Supercond. Sci. Technol. 23, 043001 (2010); M. Mondal, B. Joshi, S. Kumar, A. Kamlapure, S. C. Ganguli, A. Thamizhavel, S. S. Mandal, S. Ramakrishnan, and P. Raychaudhuri, Phys. Rev. B 86, 094520 (2012)

23) A. P. Zhuravel, B. G. Ghamsari, C. Kurter, P. Jung, S. Remillard, J. Abrahams, A. V. Lukashenko, A. V. Ustinov, and S. M. Anlage, Phys. Rev. Lett. 110, 087002 (2013).

24) H. Takeya, K. Hirata, K. Yamaura, K. Togano, M. ElMassalami, R. Rapp, F. A. Chaves, and B. Ouladdiaf, Phys. Rev. B 72, 104506 (2005); G. Eguchi, D. C. Peets, M. Kriener, S. Yonezawa, G. Bao, S. Harada, Y. Inada, G.-q. Zheng, and Y. Maeno, Phys. Rev. B 87, 161203(R) (2013).

25) Y. Maeno, S. Kittaka, T. Nomura, S. Yonezawa, and K. Ishida, J. Phys. Soc. Jpn. 81, 011009 (2012).

26) S. Ryu, A. P. Schnyder, A. Furusaki, and A. W. W. Ludwig, New J. Phys. 12, 065010 (2010).

27) A. Kitaev, Phys. Usp. 44, 131 (2001).

28) X. G. Wen and A. Zee, Phys. Rev. B 66, 235110 (2002); M. Sato, Y. Tanaka, K. Yada, and T. Yokoyama, Phys. Rev. B 83, 224511 (2011).

29) M. Sato, Phys. Rev. B 73, 214502 (2006); M. Sato and S. Fujimoto, Phys. Rev. B 79, 094504 (2009).

30) B. Béri, Phys. Rev. B 81, 134515 (2010); Y. Tanaka, T. Yokoyama, A. V. Balatsky, and N. Nagaosa, Phys. Rev. B 79, 060505(R) (2009).

31) A. P. Schnyder, P. M. R. Brydon, and C. Timm, Phys. Rev. B 85, 024522 (2012); A. P. Schnyder and S. Ryu, Phys. Rev. B 84, 060504(R) (2011).

32) M. X. Wang, Y. Xu, L. P. He, J. Zhang, X. C. Hong, P. L. Cai, Z. B. Wang, J. K. Dong, and S. Y. Li, Phys. Rev. B 93, 020503(R) (2016); W. Wei, G. J. Zhao, D. R. Kim, C. Jin, J. L. Zhang, L. Ling, L. Zhang, H. Du, T. Y. Chen, J. Zang, M. Tian, C. L. Chien, and Y. Zhang, Phys. Rev. B 94, 104503 (2016); C. Cirillo, G. Carapella, M. Salvato, R. Arpaia, M. Caputo, and C. Attanasio, Phys. Rev. B 94, 104512 (2016); G. M. Pang, M. Smidman, L. X. Zhao, Y. F. Wang, Z. F. Weng, L. Q. Che, Y. Chen, X. Lu, G. F. Chen, and H. Q. Yuan, Phys. Rev. 93, 060506 (2016); X. B. Yan, Y. Xu, L. P. He, J. K. Dong, H. Cho, D. C. Peets, J.-G. Park, and S. Y. Li, Supercond. Sci. Technol. 29, 065001 (2016); M. Isobe, M. Arai, and N. Shirakawa, Phys. Rev. B 93, 054519 (2016).

33) Y. Tanaka, Y. Mizuno, T. Yokoyama, K. Yada, and M. Sato, Phys. Rev. Lett. 105, 097002 (2010).

34) G. E. Blonder, M. Tinkham, and T. M. Klapwijk, Phys. Rev. B 25, 4515 (1982).

35) T. Yokoyama, Y. Tanaka, and J. Inoue, Phys. Rev. B 72, 220504(R) (2005).

36) R. P. Singh, A. D. Hillier, B. Mazidian, J. Quintanilla, J. F. Annett, D. McK. Paul, G. Balakrishnan, and M. R. Lees, Phys. Rev. Lett. 112, 107002 (2014); Z. F. Weng, J. L. Zhang, M. Smidman, T. Shang, J. Quintanilla, J. F. Annett, M. Nicklas, G. M. Pang, L. Jiao, W. B. Jiang, Y. Chen, F. Steglich, and H. Q. Yuan, Phys. Rev. Lett. 117, 027001 (2016). 